\title{
Shortwave emission of triboluminescence and its construct as the fluorescent light source of sustainable agriculture pests control ${ }^{*}$
}

\author{
Qiang Zhou ${ }^{\dagger}$, Yong-Jun Zheng and Xing-Xing Liu \\ College of Engineering, China Agricultural University, \\ Beijing 100083, China \\ ${ }^{\dagger}$ E-mail: zq@cau.edu.cn \\ www.cau.edu.cn
}

\begin{abstract}
Triboluminescence(TL) presents many potential applications as the dimmer light source based on its cold photon emission motivated by mechanical action. Here two shortwave TL phosphors, the Ce doped Strontium Magnesium silicate $\left(\mathrm{Sr}_{2} \mathrm{MgSi}_{2} \mathrm{O}_{7}: \mathrm{Ce}\right)$ and $\mathrm{Mn}$ coordinative methyl-triphenyl Phosphonium Bromide(MTPB: $\left.\mathrm{MnBr}_{2}\right)$, was prepared. The TL property of their formed samples were tested in the reciprocating sliding friction tester. The shortwave TLs with the peak wavelengths $444 \mathrm{~nm}$ and $517 \mathrm{~nm}$ were identified individually for inorganic phosphor $\mathrm{Sr}_{2} \mathrm{MgSi}_{2} \mathrm{O}_{7}$ :Ce and organic phosphor MTPB: $\mathrm{MnBr}_{2}$. Another a higher friction load must be set up to promote a higher TL emission in a lower phosphor wear rate. Finally a TL unit with squirrel cage structure is constructed on the basis of phosphor TL test in the sliding fiction, which supply a potential application as a fluorescent source for controlling agricultural pests without pesticides.
\end{abstract}

Keywords: Triboluminescence; Shortwave Emission; Fluorenscent source; Sliding Friction; Tribological Property; Sustainable Agriculture; Pests Control.

\section{Introduction}

Triboluminescence (TL), the light emission in the frictional process, is motivated by any mechanical action on the phosphor solids. Here the TL phosphor not only involve the inorganic compounds, but have some organic compounds[1,2]. The mechanical action may be a static contact stress to squeeze the material surface and led to the electron transition of material crystal to give out light, even is a tangential friction force to promote a relative sliding and TL excitation between contact bodies, meanwhile the wear and tribological chemistry reactions may arise on the contact surfaces[3,4], further the triboplasma will take place in the tribological process[5,6]. These all move up the complicated character of TL besides cold photon emissions.

\footnotetext{
* This work is supported by National Nature Science Fund of China (NSFC 51375482) and the Special Fund for Agro-scientific Research in the Public Interest ( SFAR-PI 201203025)..
} 
The development of TL study must suggest some applications of triboluminescence, such as stress sensor, real-time visualization monitoring of stress field in large structure, as well as novel machanoluminescence driven photocell system, and so on ${ }^{1,2}$. Another the triboluminescence as a fluorescence source may be applied to some of fields for a special illuminating application.

In this paper, the Ce doped Strontium Manganese silicate phosphorand Mn coordinative methyl-triphenyl Phosphonium Bromidephosphorwere prepared and their TL property was tested by means of reciprocating sliding friction tester; A TL unit as a light source manner for trapping agricultural pests was constituted on the basis of light emission stimulated by sliding friction.

\section{Preparation of Shortwave Triboluminescent Phosphors}

\subsection{Inorganic Phosphor Sample}

A shortwave triboluminescence phosphor of the Ce doped Strontium Magnesium silicate, $\mathrm{Sr}_{2} \mathrm{MgSi}_{2} \mathrm{O}_{7}$ : $\mathrm{Ce}$, was compound by the ratio of mole amount of folowing chemicals, $\mathrm{SrCO}_{3}, \mathrm{MgO}, \mathrm{SiO}_{2}, \mathrm{CeO}_{2}$ in the molecular structureof phosphor. Another $\mathrm{H}_{3} \mathrm{BO}_{3}$ was added to the mixture of chemicals in the ratio of $0.15 \% \mathrm{H}_{3} \mathrm{BO}_{3}$ amountto $99.85 \%$ mixture amount, as the smelting agent of mixture in the calcining; $\mathrm{CH}_{3} \mathrm{COOH}$ was also used in a little amount as a wetting agent to cohere the powder of chemicals. The cohering mixture of chemicals then was compressed from atmospheric pressure to $30 \mathrm{MPa}$ in the manual tablet press to form $\phi 70 X 6$ sample.

When the formed samples were dried in the infrared oven for ten minutes, they can be calcined in the QSX-1400 type Muffle furnace. In the calcining process, the mixture of inertgas $\mathrm{Ar}$ and Hydrogen $\mathrm{H}_{2}$ in the ratio of $95 \% \mathrm{Ar}$ to $5 \% \mathrm{H}_{2}$ must be blown into the furnace hearth to ensure the atmosphere of reduction state in the furnace hearth. Being subject to calcine up to $1200^{\circ} \mathrm{Cand}$ hold for 4 hours, the TL phosphors is obtained. Figure 1 indicates the quality phosphor samples like the disc on the corundum crucible.

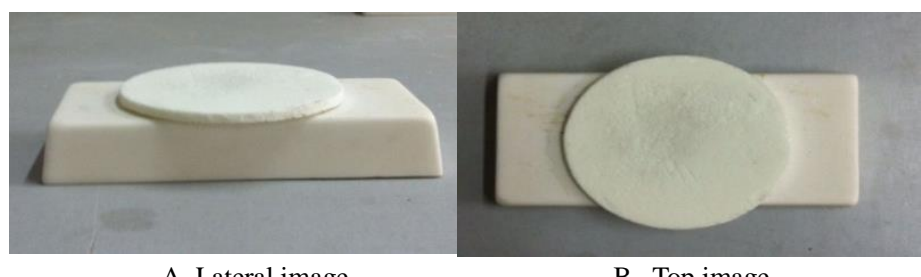

A. Lateral image $\quad$ B. Top image

Fig. 1. Sample of inorganic phosphor after calcined

\subsection{Organic Phosphor Sample}

A organic triboluminescence phosphor, $\mathrm{MnBr}_{2}$ coordinative methyl-triphenyl Phosphonium Bromide(MTPB:MnBr 2 ), short writing PMBB, was synthesized 
by $\mathrm{MnBr}_{2}$ and $\mathrm{CH}_{3}\left(\mathrm{C}_{6} \mathrm{H}_{5}\right)_{3} \mathrm{PBr}_{2}$ in the protic solvant of normal butyl alcohol in the $80^{\circ} \mathrm{C}$ temperature for 6 hours, the crystalized product of which presents the light green colore as shown in figure 2A. Similarly PMBB crystal powder was compressed from atmospheric pressure to $10 \mathrm{MPa}$ in the manual tablet press to form $\phi 70 \mathrm{X} 10$ sample as shown in figure 2B for testing triboluminescence.

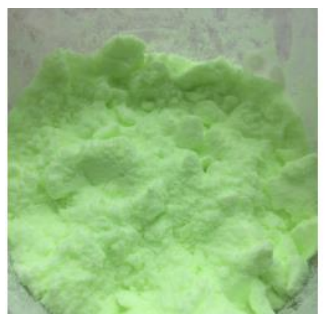

A. PMBB crystal powder

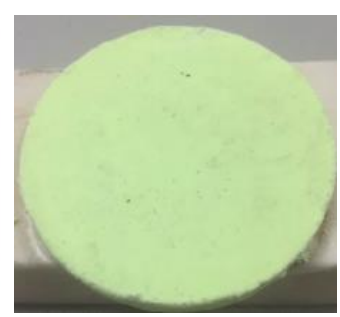

B. Formed disc sample

Fig. 2. Sample of phosphor synthesized

\section{TL Examination of Phosphor in Sliding Friction}

\subsection{Experimental Unit of Sliding Friction}

The MCJ-01A sliding friction tester with surface contact friction pair (Fig.3 A) was modified to form the linear contact friction pair that two glass bars located at the bottom on both ends of loading upper friction unit (Fig.3B) press on the formed disc of phosphor. Here a larger contact stress and a convenience space for detecting TL emission were obtained by the manner of linear contact.

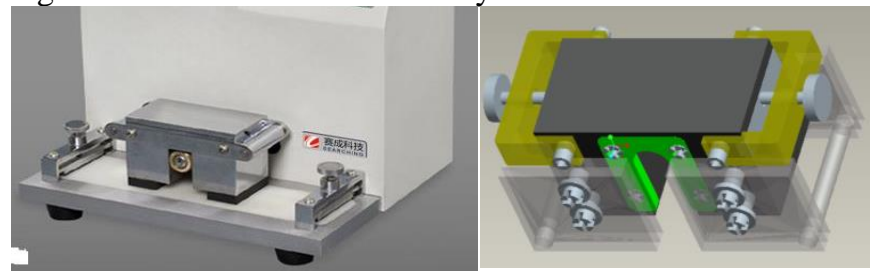

A. MCJ-01friction tester B. Design of a lineal pair

Fig. 3. MCJ-01A friction tester and its modified friction pair

The modified friction tester has a reciprocating velocity with 43 cycle per minute (cpm) and a $120 \mathrm{~mm}$ reciprocating travel. As a $\$ 70 \mathrm{X} 10$ disc sample is enough to satisfy the sliding friction of only $60 \mathrm{~mm}$ single trip. The load platform located on the upper friction unit (Fig.3B) is convenience to apply a heavier load for TL test. At same time, the optic detecting sensors connected to AvaSpecULS 2048X36 fiber optical spectrometer were mounted in the above of glass bar friction parts to measure the intensity of triboemission in the sliding friction. 


\subsection{Friction Spectrum Character of Phosphor TL}

Figure 4 presents the TL properties of $\mathrm{Sr}_{2} \mathrm{MgSi}_{2} \mathrm{O}_{7}$ :Cephosphor prepared by $1200^{\circ} \mathrm{C}$ calcining temperatures in the sliding friction of $53 \mathrm{~N}$ applied load. Obviously the prepared phosphors submits the property of shortwave emission with the peak wavelength $444 \mathrm{~nm} \pm$, full width at half maximum(FWHM)110nm and emission intensity 305 counts \pm . Meanwhile the doped Ce molar concentration in the prepared phosphors were examined, the result of which further indicates the 0.010 molar Ce doping concentration is a optimum dosage to generate a higher triboluminecent emission and smaller full width at half maximum(FWHM).

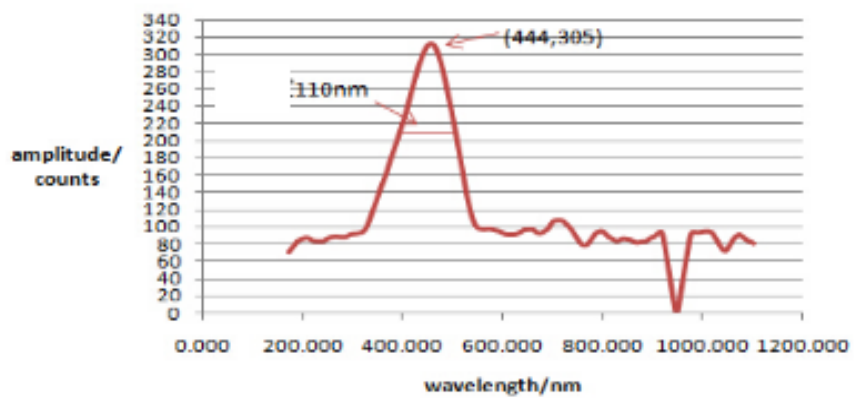

Fig. 4. TL spectrum of phosphor $\mathrm{Sr}_{2} \mathrm{MgSi}_{2} \mathrm{O}_{7}$ :Cex, $\mathrm{x}=0.010 \mathrm{~mol}$

Figure 5 indicates the TL property of MTPB:MnBr 2 phosphor in the sliding friction with applied load 46.4N. It can see that PMBB:MnBr 2 presents the green TL with $517 \mathrm{~nm}$ peak wavelength, 386 counts luminous intensity and 50nm FWHM, and the luminous spectrum possess the single peak feature.

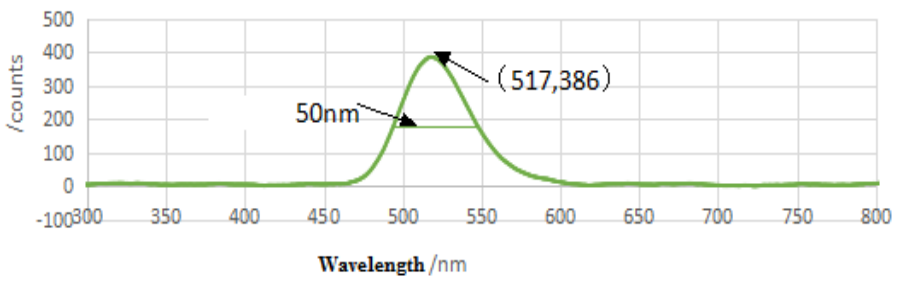

Fig. 5. TL property of PMBB phosphor

\subsection{Tribological Character of Phosphor TL.}

Here the effect of applied load on triboluminescence is investigated and the wear effect generated by applied load is tested. Figure 6,7 indicates the effects of applied load on the light emission and phosphor wear in the TL process. For $\mathrm{Sr}_{2} \mathrm{MgSi}_{2} \mathrm{O}_{7}$ :Ce and MTPB: $\mathrm{MnBr}_{2}$, the light emission intensity upgrade sharply when the applied load increases from $35 \mathrm{~N}$ to $40 \mathrm{~N}$, even $45 \mathrm{~N}$, but the wear losing rate of phosphor raises double. Therefore a suitable applied load about $40 \mathrm{~N}$ is necessary to guarantee the output of triboluminescence, dispide of inorganic 
phosphor or organic phosphor.

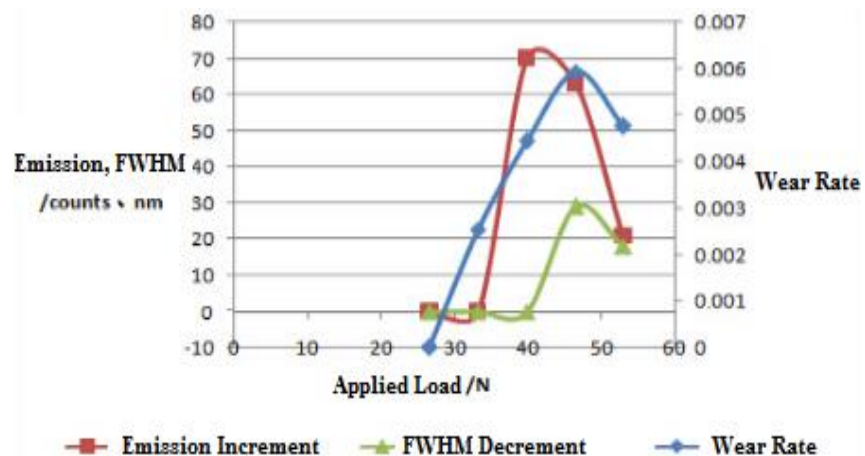

Fig. 6. Effect of applied load on $\mathrm{Sr}_{2} \mathrm{MgSi}_{2} \mathrm{O}_{7}$ :Ce phosphor property

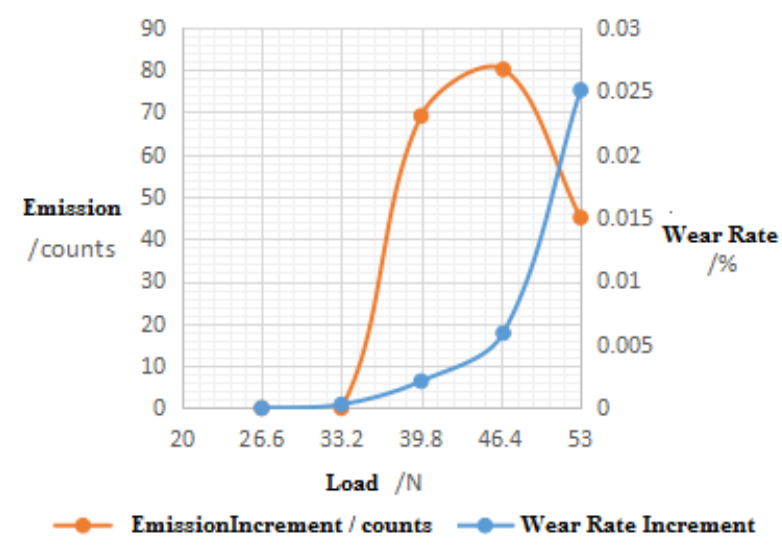

Fig. 7. Effect of applied load on MTPB:MnBr2phosphor property

\section{Construction of TL Fluorescence Source Unit}

The light source of photo taxis trapping agricultural pests usually is the shortwave fluorescent source. It supply a technological convenience for utilizing tribogical luminescent unit as photo taxis trapping light source. A squirrel cage structure is used to construct the rubbing pairs of tribological luminous unit with multiple rubbing bars against on the cylindrical phosphor coating or block, as shown in figure 8 . The unit may be mounted on the basis and was driven by means of vertical axis wind turbine connecting to the top of center axis of unit. The multiple rubbing bars against on the cylindrical phosphor can stimulate more tribological luminous flux, and the rotating TL unit also satisfy the $360^{\circ}$ azimuth light emitting, therefore it will be applied as an effective photo taxis trapping method for controlling agricultural pests without pesticides. 


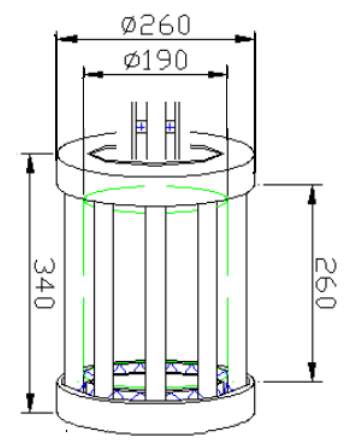

Fig. 8. Tribological luminescence pair of squirrel cage structurewith multiple rubbing bars

\section{Summary}

The shortwave TL phosphors, the Ce doped Strontium Magnesium silicate $\left(\mathrm{Sr}_{2} \mathrm{MgSi}_{2} \mathrm{O}_{7}: \mathrm{Ce}\right)$ and $\mathrm{Mn}$ coordinative methyl-triphenyl Phosphonium Bromide(MTPB: $\mathrm{MnBr}_{2}$ ), was compounded and a tablet TL phosphor sample were formed in the $\phi 70 \mathrm{X} 10$ to supply quality phosphor samples without fracture and crack for TL tests.

The TL property of phosphor samples were tested by means of reciprocating sliding friction tester and optic fiber spectrograph. The shortwave TLs with the peak wavelengths $444 \mathrm{~nm}$ and $517 \mathrm{~nm}$ were identified individually for inorganic phosphor $\mathrm{Sr}_{2} \mathrm{MgSi}_{2} \mathrm{O}_{7}: \mathrm{Ce}$ and organic phosphor MTPB: $\mathrm{MnBr}_{2}$.

A higher TL emission was obtained in both of $\mathrm{Sr}_{2} \mathrm{MgSi}_{2} \mathrm{O}_{7}: \mathrm{Ce}$ and MTPB: $\mathrm{MnBr}_{2}$ in a larger friction stress motivated by a higher friction load, hence a higher wear rate must be generated. Therefore a suitable balance between TL emission and friction load must be set up to promote TL emission in a lower wear.

The TL unit with squirrel cage structure is constructed on the basis of prepared phosphor TL test in the sliding fiction. It may supply a potential application as a fluorescent source for controlling agricultural pests without pesticides.

\section{References}

1. Piyush Jha \& B.P. Chandra. Pulse-induced mechanoluminescence of ultraviolet-irradiated SrAl2O4:Eu,Dy phosphors. Radiation Effects and Defects in Solids, 169(2014) 655-668.

2. Ross S Fontenot, William A Hollerman, Kamala N Bhat, Stephen W Allison and Mohan D Aggarwal. Luminescent properties of lanthanidedibenzoylmet hide triethylammonium compounds. Journal of Theoretical and Applied Physics,7(2013),30.

3. Changbao Han, Chi Zhang, Jingjing Tian, Xiaohui Li, Limin Zhang, Zhou $\mathrm{Li}$, and Zhonglin Wang. Triboelectrification induced by UV emission from plasmon discharge. Nano Research, 8 (2015): 219-226. 
4. Keiji Nakayama. Triboplasma Generation and Triboluminescence: Influenceof StationarySliding Partner. Tribological Letter, 37 (2010) :215228.

5. Ken'ichi Hiratsuka, Kazumasa Hosotani. Effects of friction type and humidity on triboelectrification and triboluminescenceamongeight kinds of polymers. Tribology International, 55 (2012) 87-99.

6. Shinji Muraishi, Takumi Sannomiya, Ji Shi,Yo shio Nakamura, Sirichai Leelachao.

CorrelationoftriboluminescenceandcontactstressesinZnS:Mn/polymericmatr ixcomposite Journal ofLuminescence170(2016)24-29. 